Review Article

\title{
Efficacy of Adding Bevacizumab in the First-Line Chemotherapy of Metastatic Colorectal Cancer: Evidence from Seven Randomized Clinical Trials
}

\author{
Yan-xian Chen, ${ }^{1}$ Qiong Yang, ${ }^{1}$ Jun-jie Kuang, ${ }^{1}$ Shi-yu Chen, ${ }^{2}$ \\ Ying Wei, ${ }^{1}$ Zhi-min Jiang, ${ }^{1}$ and De-rong Xie ${ }^{1}$ \\ ${ }^{1}$ Department of Oncology, Sun Yat-sen Memorial Hospital, Sun Yat-sen University, Guangzhou, Guangdong 510515, China \\ ${ }^{2}$ Department of Internal Medicine, Nanfang Hospital of Southern Medical University, Luohu District, Guangdong 510515, China
}

Correspondence should be addressed to De-rong Xie; xiederong@21cn.com

Received 14 February 2014; Revised 29 April 2014; Accepted 29 April 2014; Published 25 May 2014

Academic Editor: Antoni Castells

Copyright @ 2014 Yan-xian Chen et al. This is an open access article distributed under the Creative Commons Attribution License, which permits unrestricted use, distribution, and reproduction in any medium, provided the original work is properly cited.

\begin{abstract}
Background. Efficacy of adding bevacizumab in first-line chemotherapy of metastatic colorectal cancer (mCRC) has been controversial. The aim of this study is to gather current data to analyze efficacy of adding bevacizumab to the most used combination first-line chemotherapy in mCRC, based on the 2012 meta-analysis reported by Macedo et al. Methods. Medline, EMBASE and Cochrane library, meeting presentations and abstracts were searched. Eligible studies were randomized controlled trials (RCTs) which evaluated first-line chemotherapy with or without bevacizumab in mCRC. The extracting data were included and examined in the meta-analysis according to the type of chemotherapy regimen. Results. Seven trials, totaling 3436 patients, were analyzed. Compared with first-line chemothery alone, the adding of bevacizumab did not show clinical benefit for OS both in first-line therapy and the most used combination chemotherapy ( $\mathrm{HR}=0.89 ; 95 \% \mathrm{CI}=0.78-1.02 ; P=0.08 ; \mathrm{HR}=0.93 ; 95 \% \mathrm{CI}=0.83-$ $1.05 ; P=0.24)$. In contrast with OS, the addition of bevacizumab resulted in significant improvement for PFS $(\mathrm{HR}=0.68$; $95 \%$ $\mathrm{CI}=0.59-0.78 ; P<0.00001)$. Moreover, it also demonstrated statistical benefit for PFS in the most used combination first-line chemotherapy $(\mathrm{HR}=0.84 ; 95 \% \mathrm{CI}=0.75-0.94 ; P=0.002)$. And the subgroup analysis indicated only capacitabine-based regimens were beneficial. Conclusions. This meta-analysis shows that the addition of bevacizumab to FOLFOX/FOLFIRI/XELOX regimens might not be beneficial in terms of OS. Benefit has been seen when PFS has been taken into account. In subgroup analysis, benefit adding bevacizumab has been seen when capecitabine-based regimens are used. Further studies are warranted to explore the combination with bevacizumab.
\end{abstract}

\section{Introduction}

Colorectal cancer is the second most frequent cause of death in Europe and North America with about 600,000 deaths and a rate of approximately 1.2 million new cases every year in the whole world [1]. In order to improve the survival, more and more drugs have been introduced to the treatments of mCRC, including novel targeted drugs.

Bevacizumab, a humanized monoclonal antibody, binds to vascular endothelial growth factor (VEGF-) A, and inhibits signaling through the VEGF receptors [2]. It was approved by US Food And Drug Administration (FDA) in 2004, based on the AVF2107 study [3] comparing bolus irinotecan, 5 fluorouracil, leucovorin (IFL), and placebo therapy with IFL and bevacizumab showed a 4.7 and 4.4 month increase in median OS and PFS, respectively. However, FOLFOX, FOLFIRI [4, 5], and XELOX [6-8] became standard first-line therapy for mCRC now. Comparing FOLFOX regimen, IFL showed more early deaths, greater toxicity, and worse effectiveness in N9741 study [9]. So we explore the idea of bevacizumab added to the most used combination chemotherapy regimens-FOLFOX, FOLFIRI, and XELOX - being as beneficial as its addition to IFL regimen, as in the AVF2107 trial [3]. 
Macedo et al. [10] carried out a meta-analysis about addition of bevacizumab to first-line chemotherapy in advanced colorectal cancer, which indicated bevacizumab shows a survival benefit in $\mathrm{mCRC}$, but the current data was insufficient to support efficacy in all regimens, especially infusional fluorouracil regimens, like FOLFIRI and FOLFOX. Recently, the ITACA study, reported in ASCO meeting [11], demonstrated no statistically significant differences in PFS, OS, and ORR for adding bevacizumab to the first-line chemotherapy of mCRC.

With the advent of new trials, this meta-analysis tried to gather current data, including six RCTs from Macedo et al. meta-analysis [10] and ITACA trial [11] and then analyzed efficacy of adding bevacizumab to first-line chemotherapy or the most used combination chemotherapy of mCRC, compared with chemotherapy alone.

\section{Methods}

2.1. Search Strategy. The search strategy is similar to the one in the 2012 meta-analysis [10]. Articles published or presented from March 2011 to June 2013 were searched in the electronic databases including MEDLINE, EMBASE, and The Cochrane Library. Conference websites from ASCO, ESMO, and the World Congress on Gastrointestinal Cancer from 2011 to 2013 were also examined. An effective search strategy was performed through terms related to colorectal neoplasms, bevacizumab, and randomized controlled trials in all fields. Language was limited in English. For PubMed we used the search strategy as follows: ("Colorectal Neoplasms" [Mesh] AND "bevacizumab" [Supplementary Concept]) AND "Randomized Controlled Trials as Topic" [Mesh]. The deadline of this search was on 30 June 2013. At last, 127 potentially eligible abstracts were collected.

2.2. Selection Criteria and Quality Assessment. The studies must satisfy randomized, controlled clinical trials with a prospective design comparing first-line chemotherapy adding bevacizumab with chemotherapy alone for advanced colorectal cancer, which were previously untreated or without chemotherapy for 6 months, in case of adjuvant therapy. Those studies of poor quality (Jadad score $<3$ [17]) or involving other targeted drugs were excluded. It also should be excluded if data was not available. Methodological quality [17] of the trials was evaluated by Jadad score (range from 0 to 5) which contained randomization, masking, dropouts, and withdrawals.

2.3. Data Extraction. The main measurement outcome was OS (time from random assignment to death). The second was PFS (the time from random assignment to the first documentation of progression for disease, or death from any cause). $\mathrm{HR}$ and its 95\% confidence intervals (CI) of OS and PFS were directly collected from each selected study. When the trial had two comparative regimens, if the total HR existed, it was preferentially extracted; otherwise, the HR of each arm was selected. If data of the six primary studies reported were insufficient, they were referenced from the data shown in 2012 meta-analysis [10] which contacted corresponding authors with a request for the pending information. The following information was also extracted from each published trial: year of publication, first author, number of patients, chemotherapy regimen, follow-up period, and so forth.

The relevant articles identified from the above search strategy were assessed by two independent reviewers (Yanxian Chen and Qiong Yang). Disagreements were dealt with to achieve consensus or by a third reviewer (De-rong Xie).

2.4. Statistical Analysis and Synthesis. Statistical heterogeneity was assessed through chi-square and $I$-square test. If heterogeneity was detected ( $P$ value $<0.1$ or $I^{2}>50 \%$ ), using random model, a possible explanation for it was performed. When a reasonable cause was found, the subgroup analysis was computed. If heterogeneity was not detected, otherwise, use fixed model. OS and PFS analyses were performed using a random or fixed effects analysis model and the generic inverse variance. The results calculated were expressed by the forest plots. The effect of the treatment of each single study was expressed as a ratio of the bevacizumab-added chemotherapy regiment over chemotherapy alone. A HR value of less than one implied a clinical benefit for association of bevacizumab. A $P$ value less than 0.05 for Begg's test [18] was defined as publication bias. Meta-analyses for this study were conducted with RevMan 5.2 [19] and STATA version 12.0.

\section{Results}

3.1. Search Results. 1 new trial satisfying the above conditions was found. There were a total of 7 selected articles, when added to the previous 6 trials extracted from the 2012 metaanalysis reported by Macedo et al. [3, 11-16]. Only NO16966 study and ITACA trial contained the most used combination chemotherapy regimens. Two reviewers finally made an agreement to select these trials including 3436 patients in the meta-analysis. The flow chart is presented in Figure 1. Table 1 describes the main details of the extracted studies.

3.2. Overall Survival. Regarding OS, six studies accessed this outcome, enrolling 3314 patients. The result of the test for heterogeneity of the therapeutic effect was significant $(P=0.02)$. A random effect model was adopted. In first-line bevacizumab-added chemotherapy, compared to chemotherapy alone, the meta-analysis of data did not reach statistical significance for $\mathrm{OS}(\mathrm{HR}=0.89 ; 95 \% \mathrm{CI}=0.78-1.02$; $P=0.08$ ) (Figure 2).

We further analyzed the efficacy of adding bevacizumab in the most used combination chemotherapy. In these seven trials, only NO16966 study and ITACA trial included the most used combination chemotherapy regimens, enrolling 1777 patients. The result of the test for heterogeneity of the treatment effect was not significant $(P=0.15)$. Therefore, a fixed effects model was selected in meta-analysis. The addition of bevacizumab did not demonstrate favorable results $(\mathrm{HR}=0.93 ; 95 \% \mathrm{CI}=0.83-1.05 ; P=0.24)$ (Figure 3). Subgroup analysis by the type of fluoropyrimidine administration also did not show statistically significant data (Figure 3). 


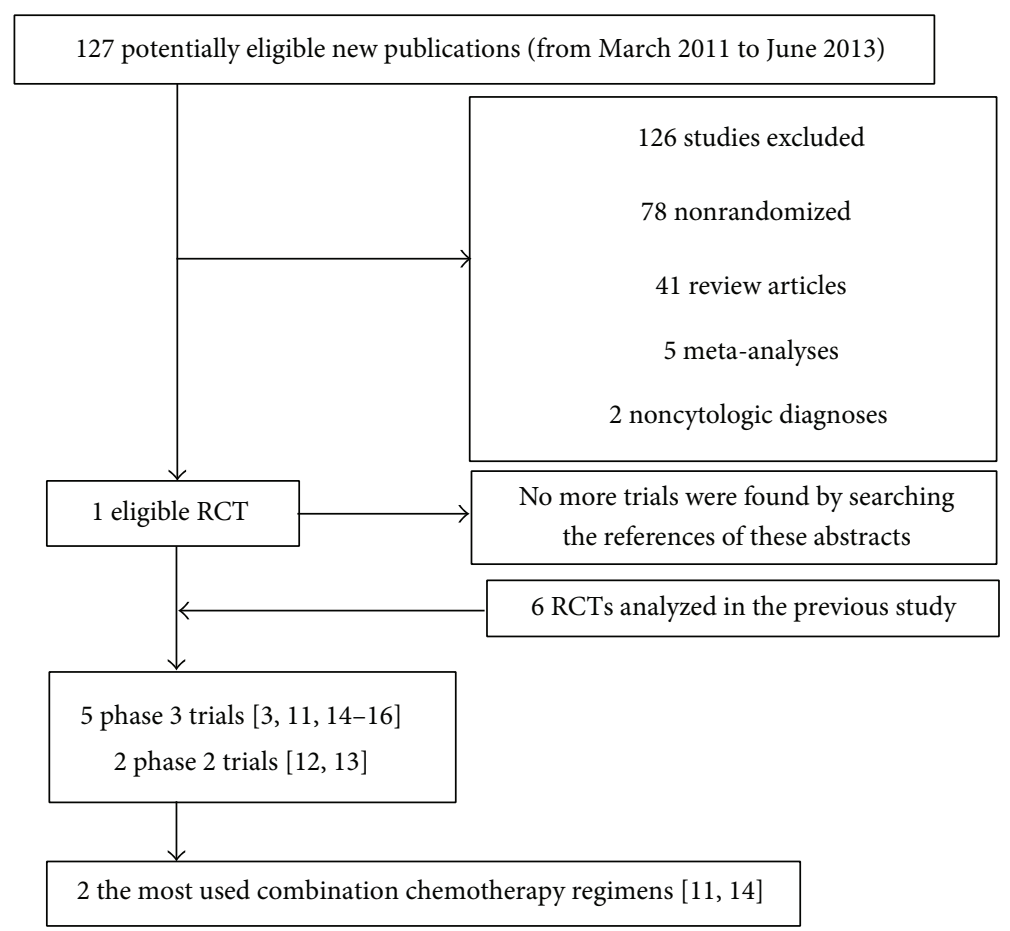

FIGURE 1: The flow chart.

\begin{tabular}{|c|c|c|c|c|c|}
\hline Study or subgroup & $\log [$ hazard ratio] & SE & Weight & $\begin{array}{c}\text { Hazard ratio } \\
\text { IV, random, } 95 \% \text { CI }\end{array}$ & $\begin{array}{c}\text { Hazard ratio } \\
\text { IV, random, } 95 \% \text { CI }\end{array}$ \\
\hline Hurwitz 2004 (AVF2107) & -0.37 & 0.08 & $18.7 \%$ & $0.69[0.59,0.81]$ & $\rightarrow$ \\
\hline Kabbinavar 2003 & 0 & 0 & & Not estimable & \\
\hline Kabbinavar 2005 & -0.24 & 0.17 & $9.7 \%$ & $0.79[0.56,1.10]$ & \\
\hline Saltz 2008 & -0.12 & 0.06 & $21.1 \%$ & $0.89[0.79,1.00]$ & - \\
\hline Serono 2013 (ITACA) & 0.17 & 0.15 & $11.3 \%$ & $1.19[0.88,1.59]$ & \\
\hline Stathopoulos 2010 & 0.05 & 0.13 & $13.1 \%$ & $1.05[0.81,1.36]$ & \\
\hline Tebutt 2010 (MAX) 1 & -0.06 & 0.13 & $13.1 \%$ & $0.94[0.73,1.22]$ & - \\
\hline Tebutt 2010 (MAX) 2 & -0.13 & 0.13 & $13.1 \%$ & $0.88[0.68,1.13]$ & \\
\hline \multirow{2}{*}{\multicolumn{5}{|c|}{$\begin{array}{l}\text { Total }(\mathbf{9 5 \%} \mathbf{C I}) \\
\text { Heterogeneity: } \tau^{2}=0.02 ; \chi^{2}=15.50, \mathrm{df}=6(P=0.02) ; I^{2}=61 \% \\
\text { Test for overall effect: } Z=1.73(P=0.08)\end{array}$}} & \\
\hline & & & & & $\begin{array}{lllll}0.5 & 0.7 & 1 & 1.5 & 2 \\
\text { OS } & \mathrm{CT}+\mathrm{B} & \mathrm{CT}\end{array}$ \\
\hline
\end{tabular}

FIGURE 2: Randomized effect model on HR of OS in first-line chemotherapy. The pooled HR of OS in first-line chemotherapy is symbolized by a solid diamond at the bottom of the forest plot and the width of which represents the $95 \%$ CI.

3.3. Progression-Free Survival. From the seven studies, one study did not offer related data. The results demonstrated an advantage to the association of bevacizumab using a random effect model $(\mathrm{HR}=0.68 ; 95 \% \mathrm{CI}=0.59-0.78$; $P<0.00001)$ compared to chemotherapy alone, though heterogeneity between trials was detected $\left(I^{2}=67 \% ; P=\right.$ 0.006) (Figure 4).

In the most used combination chemotherapy, the result of the test for heterogeneity of the treatment effect was not significant $(P=0.24)$. So, a fixed effects model was selected in meta-analysis. The analysis of the most used combination chemotherapy plus bevacizumab versus chemotherapy alone indicated uniform benefit $(\mathrm{HR}=0.84 ; 95 \%$ CI $=0.75-$ $0.94 ; P=0.002)$. The subgroup analysis showed that only capecitabine-based regimens were beneficial ( $\mathrm{HR}=0.77 ; 95 \%$ $\mathrm{CI}=0.65-0.92 ; P=0.004)$ (Figure 5).

3.4. Publication Bias. The results still did not suggest any evidence of publication bias according to the shape of funnel plots (Figures 6 and 7) or Begg's test in OS $(z=1.05, P=$ $0.293)$ and PFS $(z=-0.75, P=0.453)$.

\section{Discussion}

According to our meta-analysis, the addition of bevacizumab to first-line therapy does not prolong overall survival. HR value of the most used combination chemotherapy with adding bevacizumab rises from 0.89 to 0.93 , which has 


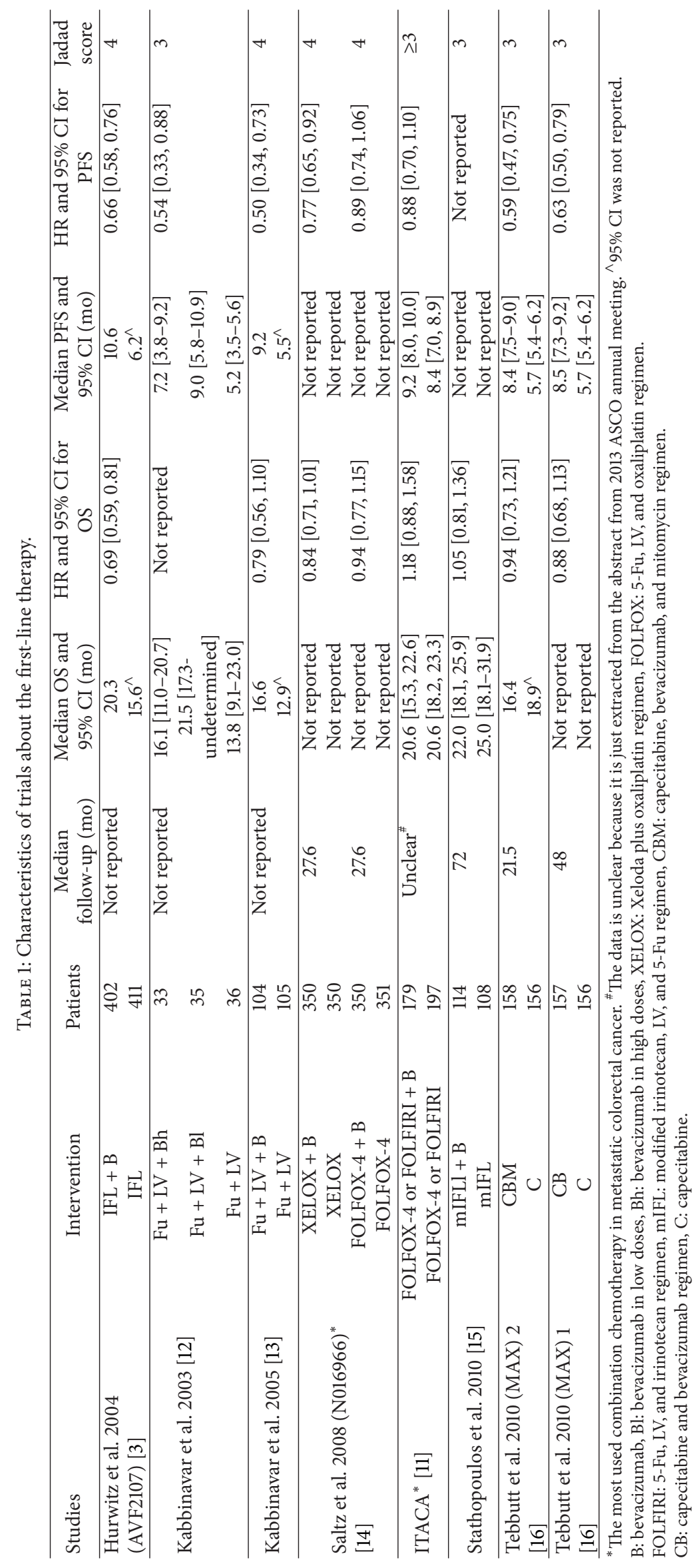




\begin{tabular}{|c|c|c|c|c|c|}
\hline Study or subgroup & $\log$ [hazard ratio] & SE & Weight & $\begin{array}{l}\text { Hazard ratio } \\
\text { IV, fixed, 95\% CI }\end{array}$ & $\begin{array}{l}\text { Hazard ratio } \\
\text { IV, fixed, } 95 \% \text { CI }\end{array}$ \\
\hline $\begin{array}{l}\text { 2.1.1 Infusional } 5 \mathrm{Fu} \\
\text { Saltz } 2008\end{array}$ & -0.06 & 0.1 & $37.3 \%$ & $0.94[0.77,1.15]$ & \\
\hline Serono 2013 (ITACA) & 0.17 & 0.15 & $16.6 \%$ & $1.19[0.88,1.59]$ & 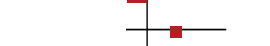 \\
\hline Subtotal $(95 \% \mathrm{CI})$ & & & $53.9 \%$ & $1.01[0.86,1.19]$ & \\
\hline \multicolumn{6}{|c|}{ Heterogeneity: $\chi^{2}=1.63, \mathrm{df}=1(P=0.20) ; I^{2}=39 \%$} \\
\hline \multicolumn{6}{|c|}{ Test for overall effect: $Z=0.13(P=0.90)$} \\
\hline \multicolumn{6}{|c|}{ 2.1.2 Capecitabine-based regimens } \\
\hline Saltz 2008 & -0.17 & 0.09 & $46.1 \%$ & $0.84[0.71,1.01]$ & \\
\hline Subtotal $(95 \% \mathrm{CI})$ & & & $46.1 \%$ & $0.84[0.71,1.01]$ & \\
\hline \multicolumn{6}{|c|}{ Heterogeneity: not applicable } \\
\hline \multicolumn{6}{|c|}{ Test for overall effect: $Z=1.89(P=0.06)$} \\
\hline Total $(95 \% \mathrm{CI})$ & & & $100.0 \%$ & $0.93[0.83,1.05]$ & \\
\hline \multicolumn{6}{|c|}{ Heterogeneity: $\chi^{2}=3.80, \mathrm{df}=2(P=0.15) ; I^{2}=47 \%$} \\
\hline \multicolumn{3}{|c|}{ Test for overall effect: $Z=1.19(P=0.24)$} & \multicolumn{2}{|c|}{ Test for subgroup differences: $\chi^{2}=2.18, \mathrm{df}=1(P=0.14), I^{2}=54.0 \%$} & $\begin{array}{c}0.50 .7 \quad 1 \quad 1.52 \\
\text { CT + B CT }\end{array}$ \\
\hline
\end{tabular}

FIGURE 3: Fixed effect model on HR of OS in the most used combination chemotherapy. The pooled HR of OS in standard first-line chemotherapy is symbolized by a solid diamond at the bottom of the forest plot and the width of which represents the $95 \%$ CI.

\begin{tabular}{|c|c|c|c|c|c|}
\hline Study or subgroup & $\log [$ hazard ratio] & SE & Weight & $\begin{array}{c}\text { Hazard ratio } \\
\text { IV, random, } 95 \% \text { CI }\end{array}$ & $\begin{array}{c}\text { Hazard ratio } \\
\text { IV, random, } 95 \% \text { CI }\end{array}$ \\
\hline Hurwitz 2004 (AVF2107) & -0.41 & 0.07 & $19.8 \%$ & $0.66[0.58,0.76]$ & $-\square$ \\
\hline Kabbinavar 2003 & -0.62 & 0.25 & $6.3 \%$ & $0.54[0.33,0.88]$ & \\
\hline Kabbinavar 2005 & -0.69 & 0.19 & $9.2 \%$ & $0.50[0.35,0.73]$ & \\
\hline Saltz 2008 & -0.19 & 0.06 & $20.8 \%$ & $0.83[0.74,0.93]$ & $\rightarrow-$ \\
\hline Serono 2013 (ITACA) & -0.13 & 0.12 & $14.6 \%$ & $0.88[0.69,1.11]$ & 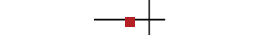 \\
\hline Stathopoulos 2010 & 0 & 0 & & Not estimable & \\
\hline Tebutt 2010 (MAX) 1 & -0.52 & 0.12 & $14.6 \%$ & $0.59[0.47,0.75]$ & 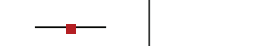 \\
\hline Tebutt 2010 (MAX) 2 & -0.46 & 0.12 & $14.6 \%$ & $0.63[0.50,0.80]$ & $\longrightarrow$ \\
\hline \multicolumn{5}{|c|}{$\begin{array}{lll}\text { Total }(95 \% \mathrm{CI}) & 100.0 \% & 0.68[0.59,0.78]\end{array}$} & \\
\hline \multicolumn{6}{|c|}{$\begin{array}{l}\text { Heterogeneity: } \tau^{2}=0.02 ; \chi^{2}=18.14, \mathrm{df}=6(P=0.006) ; I^{2}=67 \% \\
\text { Test for overall effect: } Z=5.28(P<0.00001)\end{array}$} \\
\hline
\end{tabular}

FIGURE 4: Randomized effect model on HR of PFS in first-line chemotherapy. The beneficial HR of PFS in standard first-line chemotherapy is symbolized by a solid diamond at the bottom of the forest plot and the width of which represents the 95\% CI.

a much narrower 95\% confidence interval (from 95\% CI = $0.78-1.02$ to $95 \% \mathrm{CI}=0.83-1.05)$ and better homogeneity (from $I^{2}=61 \%, P=0.02$ to $I^{2}=47 \% ; P=0.15$ ), suggesting the result of no survival benefit regards to OS is more powerful.

Regarding PFS, however, it appears to be advantageous in both first-line therapy and the most used combination firstline chemotherapy. To further analyze, it is the XELOX subgroup that shows the benefit, supported by the single-study NO16966 [14]. In contrast to the XELOX subset, infusional 5Fu regimens, like FOLFOX or FOLFIRI, do not prolong PFS. The administration and the drug type of fluoropyrimidine might be the main reason for the distinct therapeutic effect of bevacizumab addition to different fluoropyrimidine-based chemotherapy regimens.

The addition of bevacizumab to first-line chemotherapy in mCRC is still a paradigm left unsolved. Compared with
2012 meta-analysis, adding the ITACA study [11] which only takes the weight of $11.3 \%$ in all enrolled trials, the entire consequence of this meta-analysis is quite different regarding OS. It means that a survival benefit of the 2012 meta-analysis is not obvious, and the conclusion is very unsound. But for PFS, the result is quite similar. Addition of bevacizumab to infusional 5-Fu-based regimens, like FOLFOX or FOLFIRI, does not show clinical benefit, while adding bevacizumab to capacitabine-based regimens are beneficial. It may be instrumental to find which chemotherapy regimen is effective associated with bevacizumab by comparing the clinical benefit between bevacizumab and unhomogeneous fluorouracil agents.

The benefit of PFS cannot turn into OS so far according to the above results. Firstly, of the seven selected trials, three made PFS as primary endpoint for original design, especial NO16966 study [14] and ITACA trial [11]. However, 


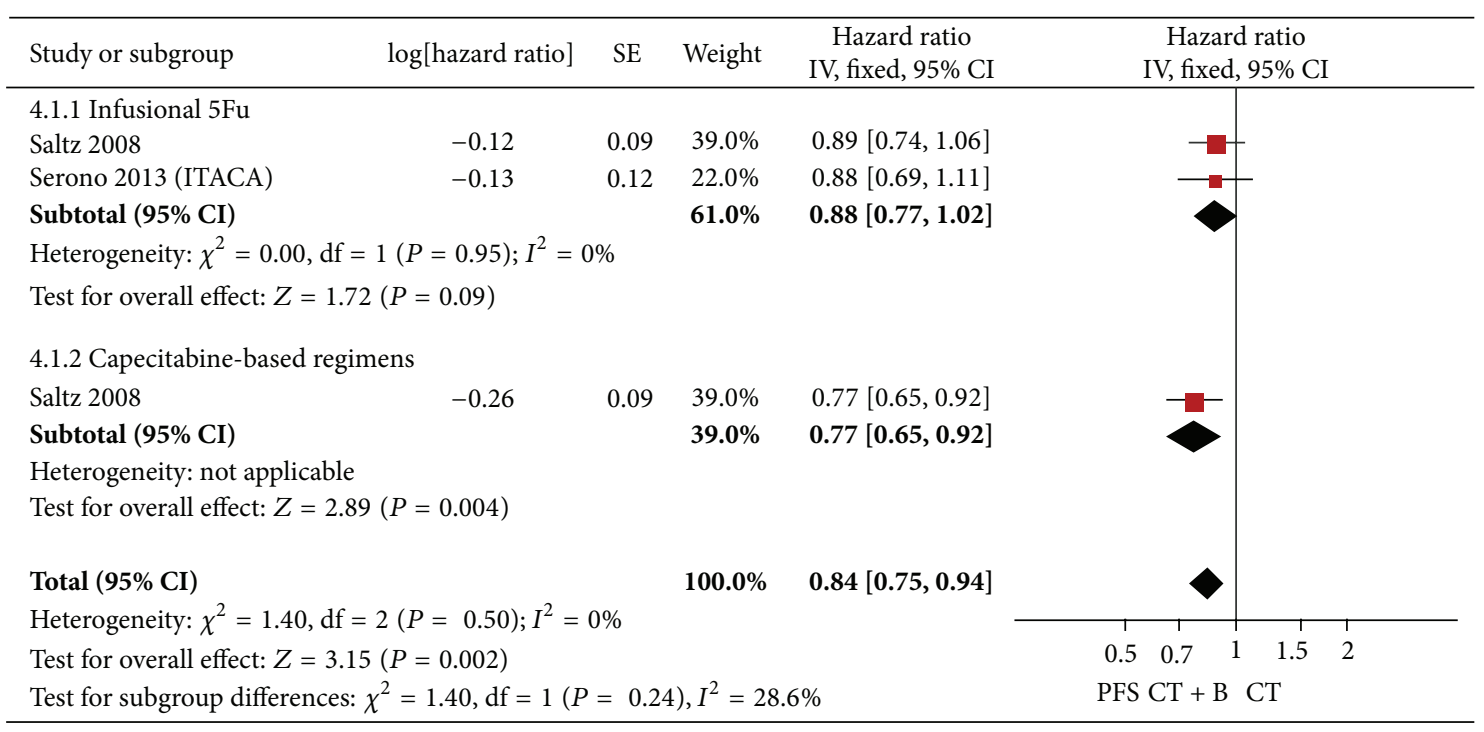

FIGURE 5: Fixed effect model on HR of PFS in the most used combination chemotherapy. The beneficial HR of PFS in standard first-line chemotherapy is symbolized by a solid diamond at the bottom of the forest plot and the width of which represents the $95 \%$ CI.

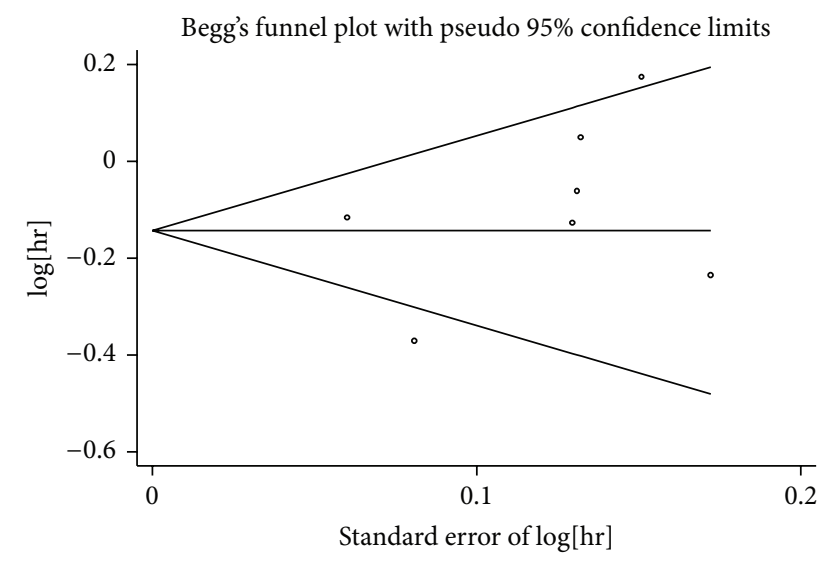

FIGURE 6: Funnel plot for publication bias test OS. The two oblique lines indicate the pseudo $95 \%$ confidence limits.

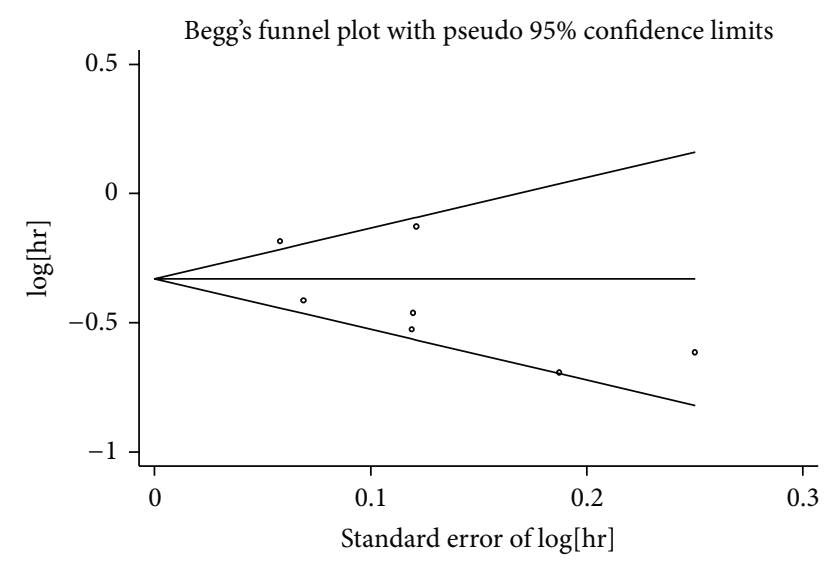

FIGURE 7: Funnel plot for publication bias test PFS. The two oblique lines indicate the pseudo $95 \%$ confidence limits. we choose OS as the main measurement outcome in this meta-analysis. Thus, the distinct experimental design may be the reason why the benefit of PFS cannot turn into OS. In addition, the current data proves that bevacizumab plus chemotherapy (crossed over from first-line regimen) which continued beyond progression significantly prolonged OS and PFS (1.4 months, 1.6 months, resp.) in second-line mCRC in ML18147 study [20]. But, the treatment of combination with bevacizumab is limited to the first-line in all enrolled trials, without considering the effect of the second- and further-line regimen on OS. Moreover, preliminary data suggests that there are some groups that might benefit more from the combination of bevacizumab, but the included studies of our meta-analysis cannot explore these factors, just like patients with primary tumors originating in the rectum and sigmoid colon [21], or certain targets like wild-type KRAS [22] and mismatch repair defective (dMMR) tumors [23], or the combination with certain drugs for medication interactions. Further studies are required to confirm the findings.

Several limitations have to be mentioned regarding this meta-analysis. One is that all of the data are extracted from abstracted data (AD) and not individual patient data (IPD), which would be less convincing to confirm the results. But, a correlation analysis shows AD meta-analysis is strongly associated with IPD meta-analysis [24]. It means AD is a kind of acceptable and practical method of meta-analysis alternative for IPD. Another limitation is that we chose OS as the main endpoint instead of PFS which was the primary endpoint originally in most trials. As we all know, OS is a more objective index and an ending point in the field of incurable diseases like $\mathrm{mCRC}$, and it needs to enroll more cases in the trial. However PFS may be influenced by the sensitivity of imaging instrument, experience of checker, and timeliness discovery of the tumor progression. In addition, the studies enroll 1777 patients in the most used combination 
chemotherapy regimens. They are too few to carry out more subgroups analysis. Only ITACA study contained FOLFIRI regimen. However, it cannot perform subsets analysis because we cannot obtain more information about bevacizumab addition to FOLFIRI or FOLFOX regimen from the ITACA abstract reported in the 2013 ASCO meeting.

\section{Conclusion}

This meta-analysis shows that the addition of bevacizumab to FOLFOX/FOLFIRI/XELOX regimens might not be beneficial in terms of OS, compared to chemotherapy alone. Benefit has been seen when PFS has been taken into account. In subgroup analysis, benefit of adding bevacizumab has been seen when capecitabine-based regimens are used. Further studies are warranted to explore the combination with bevacizumab.

\section{Conflict of Interests}

The authors have declared that there is no conflict of interests regarding the publication of this paper.

\section{Authors' Contribution}

Yan-xian Chen and Qiong Yang contributed equally to this paper.

\section{Acknowledgment}

The authors of this paper would like to thank the authors who kindly provided the data necessary for the study.

\section{References}

[1] http://globocan.iarc.fr/Default.aspx.

[2] D. J. Hicklin and L. M. Ellis, "Role of the vascular endothelial growth factor pathway in tumor growth and angiogenesis," Journal of Clinical Oncology, vol. 23, no. 5, pp. 1011-1027, 2005.

[3] H. Hurwitz, L. Fehrenbacher, W. Novotny et al., "Bevacizumab plus irinotecan, fluorouracil, and leucovorin for metastatic colorectal cancer," The New England Journal of Medicine, vol. 350, no. 23, pp. 2335-2342, 2004.

[4] C. Tournigand, T. André, E. Achille et al., "FOLFIRI followed by FOLFOX6 or the reverse sequence in advanced colorectal cancer: a randomized GERCOR study," Journal of Clinical Oncology, vol. 22, no. 2, pp. 229-237, 2004.

[5] G. Colucci, V. Gebbia, G. Paoletti et al., "Phase III randomized trial of FOLFIRI versus FOLFOX4 in the treatment of advanced colorectal cancer: A Multicenter Study of the Gruppo Oncologico Dell'Italia Meridionale," Journal of Clinical Oncology, vol. 23, no. 22, pp. 4866-4875, 2005.

[6] J. Cassidy, S. Clarke, E. Díaz-Rubio et al., "Randomized phase III study of capecitabine plus oxaliplatin compared with fluorouracil/folinic acid plus oxaliplatin as first-line therapy for metastatic colorectal cancer," Journal of Clinical Oncology, vol. 26, no. 12, pp. 2006-2012, 2008.

[7] J. Cassidy, J. Tabernero, C. Twelves et al., "XELOX (capecitabine plus oxaliplatin): active first-line therapy for patients with metastatic colorectal cancer," Journal of Clinical Oncology, vol. 22, no. 11, pp. 2084-2091, 2004.
[8] R. Porschen, H.-T. Arkenau, S. Kubicka et al., "Phase III study of capecitabine plus oxaliplatin compared with fluorouracil and leucovorin plus oxaliplatin in metastatic colorectal cancer: a final report of the AIO colorectal study group," Journal of Clinical Oncology, vol. 25, no. 27, pp. 4217-4223, 2007.

[9] R. M. Goldberg, D. J. Sargent, R. F. Morton et al., "A randomized controlled trial of fluorouracil plus leucovorin, irinotecan, and oxaliplatin combinations in patients with previously untreated metastatic colorectal cancer," Journal of Clinical Oncology, vol. 22, no. 1, pp. 23-30, 2004.

[10] L. T. Macedo, A. B. C. Lima, and A. D. Sasse, "Addition of bevacizumab to first-line chemotherapy in advanced colorectal cancer: a systematic review and meta-analysis, with emphasis on chemotherapy subgroups," BMC Cancer, vol. 12, article 89, 2012.

[11] A. Passardi, E. Scarpi, L. Cavanna et al., "Effectiveness of bevacizumab added to gold standard chemotherapy in metastatic colorectal cancer (mCRC): final results from the Itaca randomized clinical trial," Journal of Clinical Oncology, vol. 31, supplement 15, p. 3517, 2013.

[12] F. Kabbinavar, H. I. Hurwitz, L. Fehrenbacher et al., "Phase II, randomized trial comparing bevacizumab plus fluorouracil (FU)/leucovorin (LV) with FU/LV alone in patients with metastatic colorectal cancer," Journal of Clinical Oncology, vol. 21, no. 1, pp. 60-65, 2003.

[13] F. F. Kabbinavar, J. Schulz, M. McCleod et al., "Addition of bevacizumab to bolus fluorouracil and leucovorin in first-line metastatic colorectal cancer: results of a randomized phase II trial," Journal of Clinical Oncology, vol. 23, no. 16, pp. 3697-3705, 2005.

[14] L. B. Saltz, S. Clarke, E. Díaz-Rubio et al., "Bevacizumab in combination with oxaliplatin-based chemotherapy as first-line therapy in metastatic colorectal cancer: a randomized phase III study," Journal of Clinical Oncology, vol. 26, no. 12, pp. 20132019, 2008.

[15] G. P. Stathopoulos, C. Batziou, D. Trafalis et al., "Treatment of colorectal cancer with and without bevacizumab: a phase III study," Oncology, vol. 78, no. 5-6, pp. 376-381, 2010.

[16] N. C. Tebbutt, K. Wilson, V. J. Gebski et al., "Capecitabine, bevacizumab, and mitomycin in first-line treatment of metastatic colorectal cancer: results of the Australasian Gastrointestinal Trials Group randomized phase III MAX Study," Journal of Clinical Oncology, vol. 28, no. 19, pp. 3191-3198, 2010.

[17] A. R. Jadad, R. A. Moore, D. Carroll et al., "Assessing the quality of reports of randomized clinical trials: is blinding necessary?" Controlled Clinical Trials, vol. 17, no. 1, pp. 11-12, 1996.

[18] J. A. C. Sterne, M. Egger, and G. D. Smith, "Systematic reviews in health care: investigating and dealing with publication and other biases in meta-analysis," British Medical Journal, vol. 323, no. 7304, pp. 101-105, 2001.

[19] Cochrane IMS, http://tech.cochrane.org/revman/download.

[20] J. Bennouna, J. Sastre, D. Arnold et al., "Continuation of bevacizumab after first progression in metastatic colorectal cancer (ML18147): a randomised phase 3 trial," Lancet Oncology, vol. 14, no. 1, pp. 29-37, 2013.

[21] M. K. Boisen, J. S. Johansen, C. Dehlendorff et al., "Primary tumor location and bevacizumab effectiveness in patients with metastatic colorectal cancer," Annals of Oncology, vol. 24, no. 10, pp. 2554-2559, 2013.

[22] F. Petrelli, A. Coinu, M. Cabiddu et al., "KRAS as prognostic biomarker in metastatic colorectal cancer patients treated with bevacizumab: a pooled analysis of 12 published trials," Medical Oncology, vol. 30, no. 3, p. 650, 2013. 
[23] K. Pogue-Geile, G. Yothers, Y. Taniyama et al., "Defective mismatch repair and benefit from bevacizumab for colon cancer: findings from NSABP C-08," Journal of the National Cancer Institute, vol. 105, no. 13, pp. 989-992, 2013.

[24] E. Bria, R. J. Gralla, H. Raftopoulos, and D. Giannarelli, "Comparing two methods of meta-analysis in clinical research-individual patient data-based (IPD) and literaturebased abstracted data (AD) methods: analyzing five oncology issues involving more than 10,000 patients in randomized clinical trials (RCTs)," Journal of Clinical Oncology, vol. 25, no. 18, supplement, abstract 6512, 2007. 


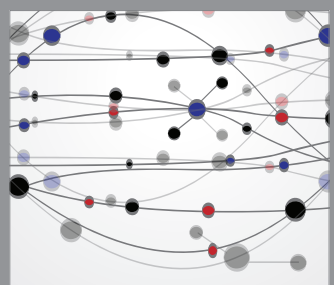

The Scientific World Journal
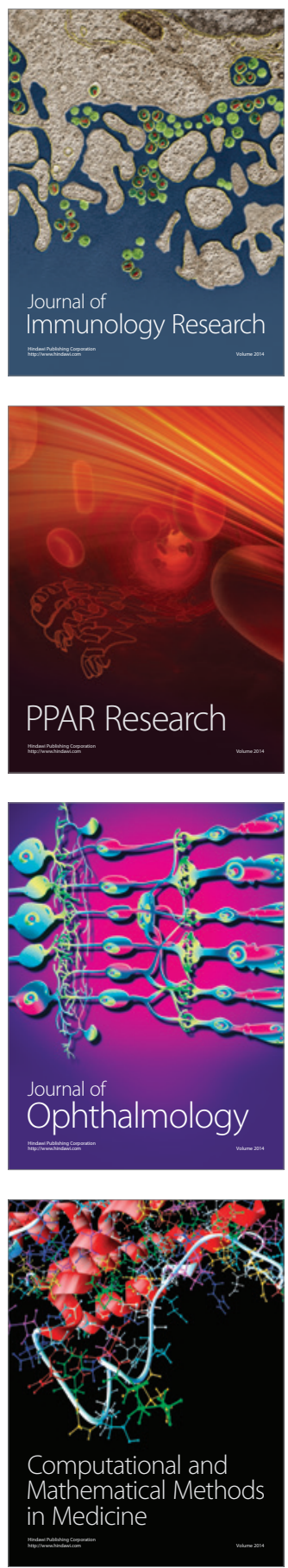

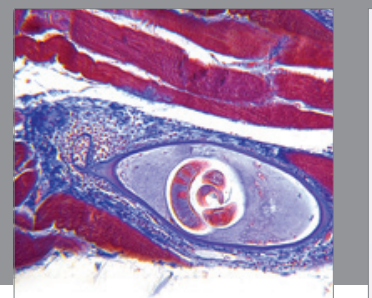

Gastroenterology

Research and Practice
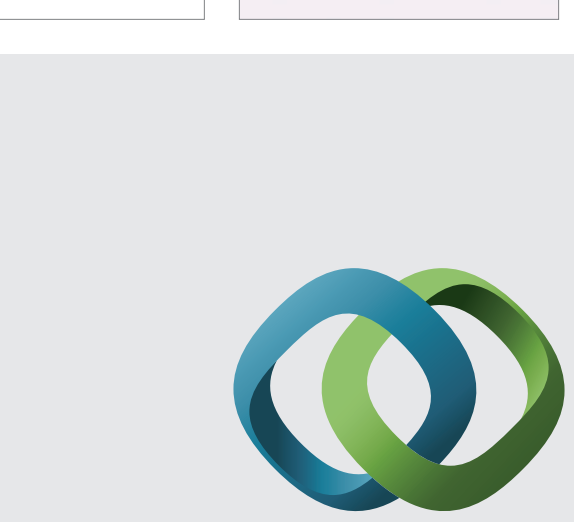

\section{Hindawi}

Submit your manuscripts at

http://www.hindawi.com
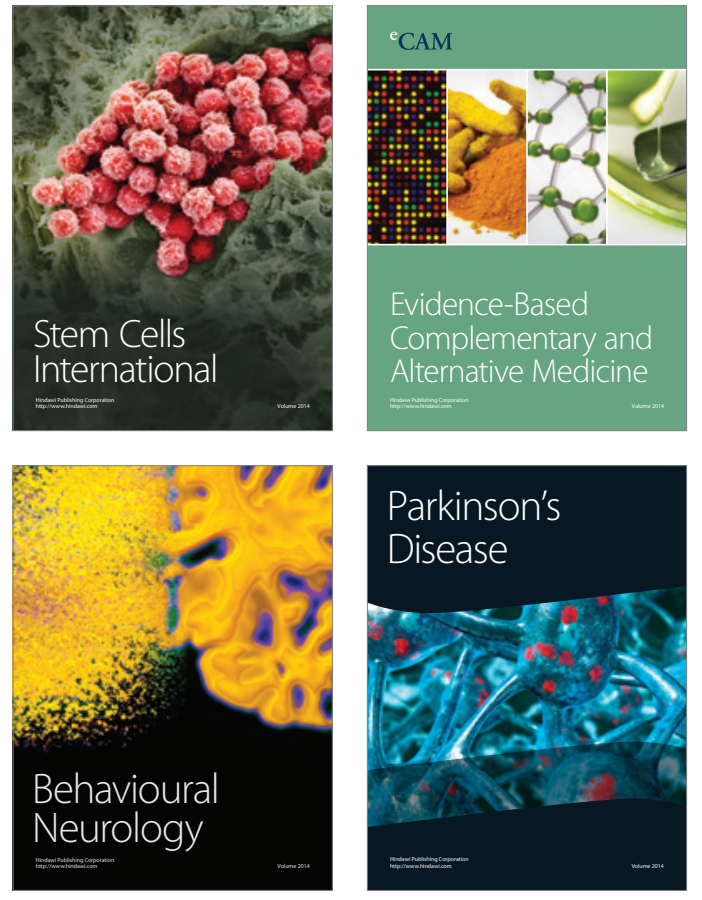
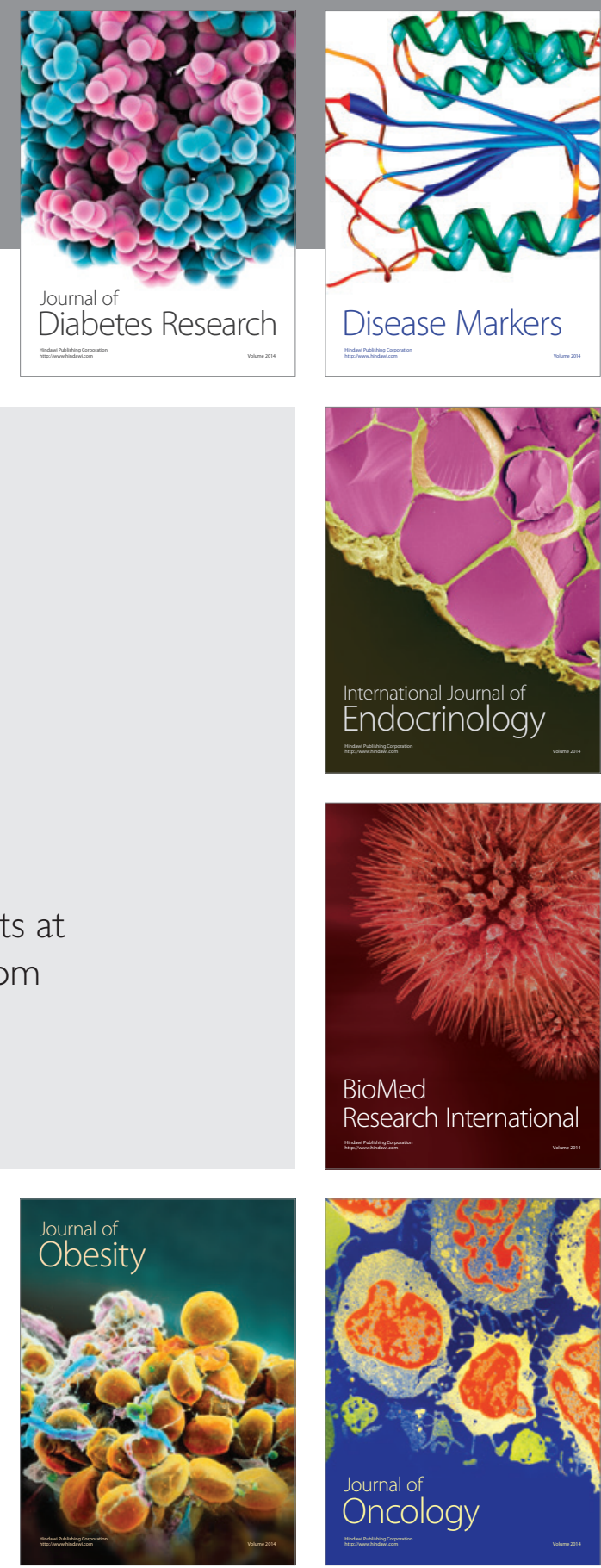

Disease Markers
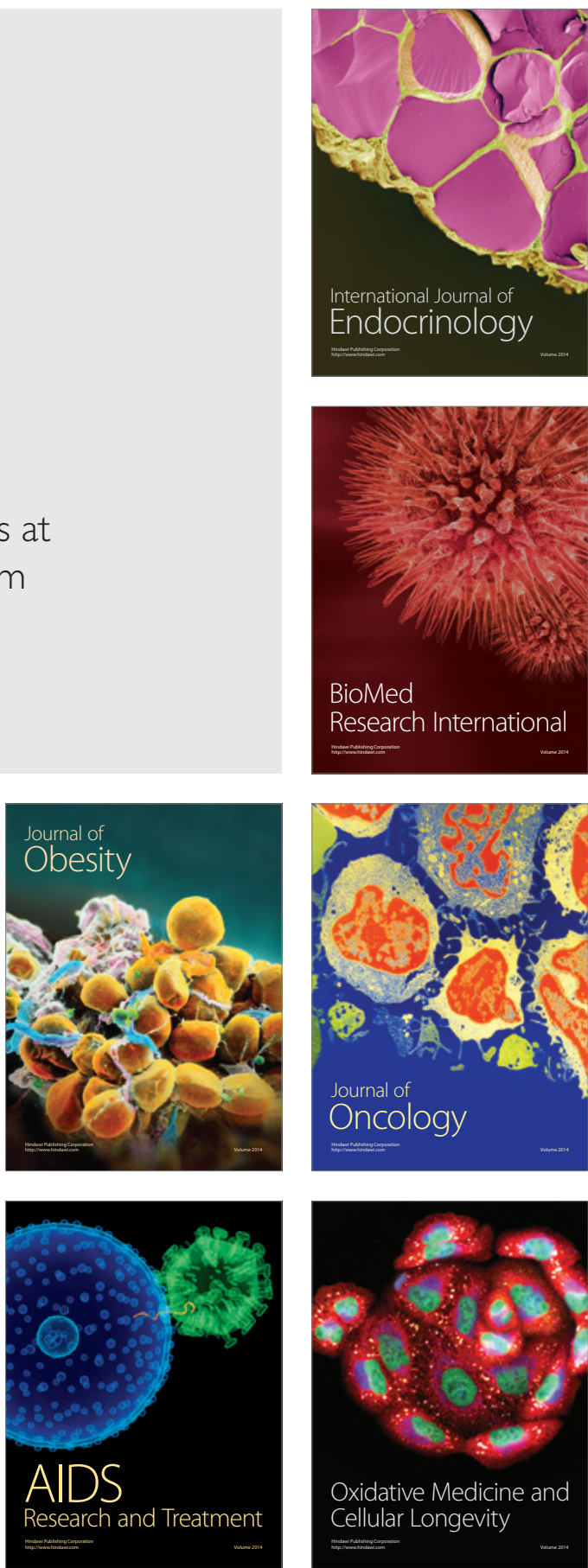